\title{
Technologies for Social Augmentations in User-Embodied Virtual Reality
}

\author{
Daniel Roth \\ daniel.roth@uni-wuerzburg.de \\ University of Würzburg, HCI Group \\ Würzburg, Germany
}

\author{
Gary Bente \\ Michigan State University \\ East Lansing, USA
}

\author{
Peter Kullmann \\ David Mal \\ Chris Felix Purps \\ University of Würzburg, HCI Group
}

\author{
Kai Vogeley \\ University Hospital Cologne \\ Cologne, Germany
}

Marc Erich Latoschik

University of Würzburg, HCI Group

Würzburg, Germany
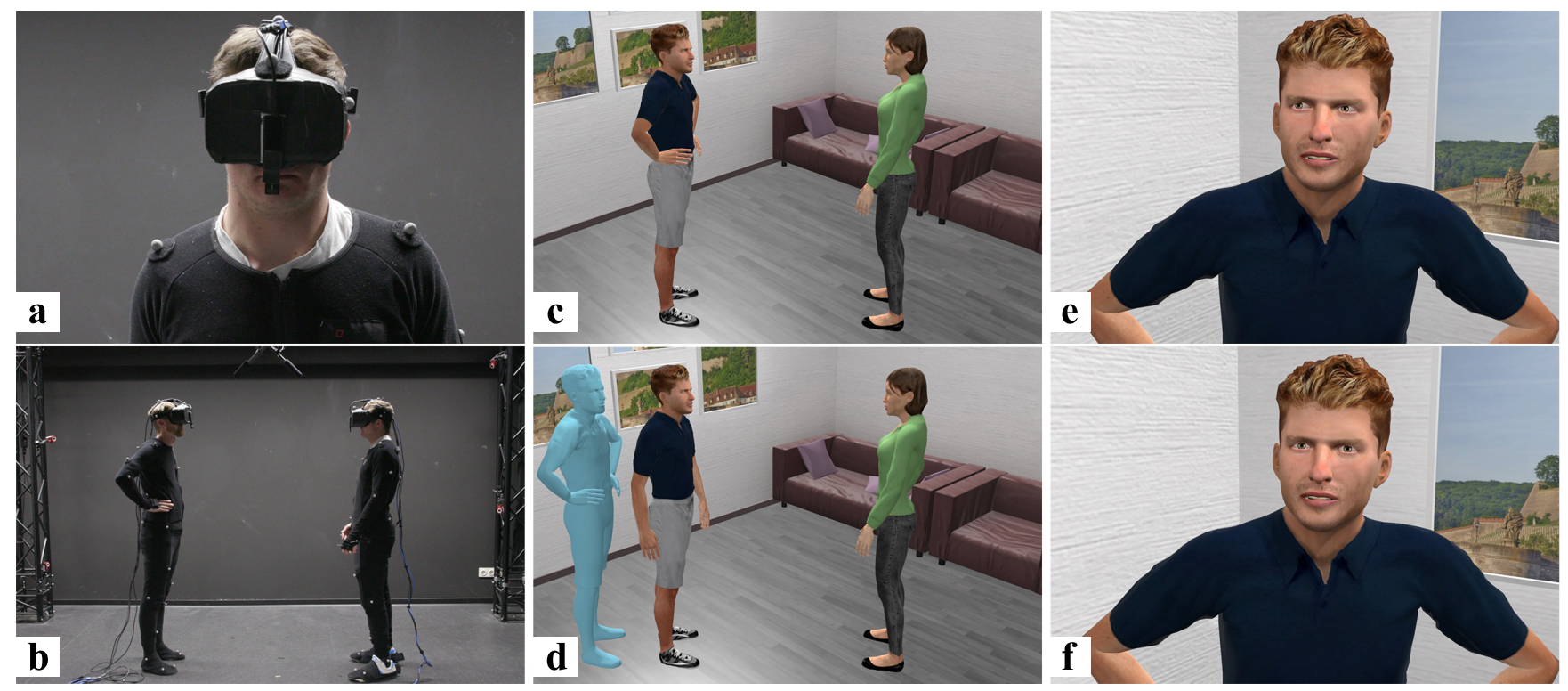

Figure 1: Prototype. Body motion, facial expressions and gaze replicated and augmented in immersive social interaction. $a, b$ ) Apparatus for behavior sensing. c) Behaviors replicated to avatars that can be flexible in their appearance. $d$ ) Artificial mimicry (vs. original behavior: blue mannequin). $e$ ) Original eye tracking data, and f) artificially directed gaze.

\section{ABSTRACT}

Technologies for Virtual, Mixed, and Augmented Reality (VR, MR, and AR) allow to artificially augment social interactions and thus to go beyond what is possible in real life. Motivations for the use of social augmentations are manifold, for example, to synthesize behavior when sensory input is missing, to provide additional affordances in shared environments, or to support inclusion and training of individuals with social communication disorders. We review and categorize augmentation approaches and propose a software architecture based on four data layers. Three components further handle the status analysis, the modification, and the blending of

Permission to make digital or hard copies of part or all of this work for personal or classroom use is granted without fee provided that copies are not made or distributed for profit or commercial advantage and that copies bear this notice and the full citation on the first page. Copyrights for third-party components of this work must be honored. For all other uses, contact the owner/author(s).

VRST '19, November 12-15, 2019, Parramatta, NSW, Australia

(C) 2019 Copyright held by the owner/author(s).

ACM ISBN 978-1-4503-7001-1/19/11.

https://doi.org/10.1145/3359996.3364269 behaviors. We present a prototype (injectX) that supports behavior tracking (body motion, eye gaze, and facial expressions from the lower face), status analysis, decision-making, augmentation, and behavior blending in immersive interactions. Along with a critical reflection, we consider further technical and ethical aspects.

\section{CCS CONCEPTS}

- Human-centered computing $\rightarrow$ Virtual reality; Mixed/augmented reality; • Software and its engineering;

\section{KEYWORDS}

augmented social interaction, virtual reality, artificial intelligence ACM Reference Format:

Daniel Roth, Gary Bente, Peter Kullmann, David Mal, Chris Felix Purps, Kai Vogeley, and Marc Erich Latoschik. 2019. Technologies for Social Augmentations in User-Embodied Virtual Reality. In 25th ACM Symposium on Virtual Reality Software and Technology (VRST '19), November 12-15, 2019, Parramatta, NSW, Australia. ACM, New York, NY, USA, 12 pages. https://doi.org/10.1145/3359996.3364269 


\section{INTRODUCTION}

Communication is one of the decisive characteristics that define human nature [Tomasello 2009], and social interaction through adaptive nonverbal behavior is a crucial element of our everyday life that begins developing right away, as infants [Cappella 1981]. Systems that support social interaction in VR usually strive for the realistic replication of behavior and appearance. In their $1992 \mathrm{CHI}$ paper Hollan and Stornetta described their review of the guiding principle of communication media development: "A belief in the efficacy of imitating face-to-face communication is an unquestioned presupposition of most current work on supporting communications in electronic media" [Hollan and Stornetta 1992, p. 119], and they go on and state that "looking at non-imitative approaches that focus on underlying explored requirements and the distinctive characteristics of the electronic media rather than on imitation of the mechanisms of face-to-face might lead to even better solutions" [Hollan and Stornetta 1992, p. 124]. The latter specifically addresses VR systems, which have an even greater potential to simulate the artificial rather than replicate the real, and, in consequence, to change the way we communicate. Multiple previous studies have explored the adaptation of a virtual body [Slater et al. 2010], and argued for the human flexibility to adapt non-human avatars [Won et al. 2015]. User embodiment, in turn, can lead to higher social presence ratings, comparable to face-to-face interactions [Smith and Neff 2018]. With regard to augmented communication in virtual environments, Gerhard et al. [2001] explored hybrid human/agent communication models to allow for continuous presence in educational virtual environments. Furthermore, Bailenson et al. [2004] explored the transformation and decoupling of virtual behaviour from its physical counterpart in 2004. Roth et al. [2015] introduced the concept of hybrid avatar-agent technology (HAAT) to augment virtual encounters. Through ambient sensing, ubiquitous computing, and the omnipresence of display technology, the flexibility of virtual communication will heavily impact the way we interact and thus affect society as a whole.

In this regard, the exploration of social augmentations can lead to a number of benefits, including 1) a deeper understanding of virtual communicative processes, 2) resolving a lack of sensory channels as well as transmission errors based on a reconstruction through social behavior models, and 3) the fostering of the inclusion of users with impairments in communication and/or interaction who cannot interpret and/or express nonverbal behavior.

\subsection{Contributions}

We present a system architecture for the augmentation of social behaviors in multi-user environments. The architecture merges avatar-mediated behavior controlled by humans with agent-based behavior controlled by the simulation, guided by a social intelligence to govern and transform interactions based on the detection of triggers. We present a prototype (see Fig. 1) and evaluate its functionalities. To the best of our knowledge, the present system is the first immersive multi-user system that supports head-mounted display (HMD) based embodied interactions replicating body motions, facial expressions, and gaze behaviors to avatars and the first system that allows for a manifold modification of such behaviors.

\section{RELATED WORK}

\subsection{Social Interaction in Virtual Environments}

In 1990, Blanchard et al. [1990] created RB2 ("reality built for 2"), a simulation rendered through "eyephones" (today: HMD) that supported gestural interaction via tracking gloves and body sensing. Systems that allow for multi-user interaction can be categorized in one of two main approaches: i) Telepresence systems that build upon Minsky's idea of telepresence [Minsky 1980] in the context of communication [Beck et al. 2013], which typically involves camera-based real-time 3D capturing and reconstruction of human users, and ii) Avatar-mediated systems based on avatars, computergenerated virtual characters that are driven by tracking human behavior [Bailenson and Blascovich 2004]. In general, these systems allow for user embodiment, meaning the "provision of users with appropriate body images so as to represent them to others (and also to themselves) in collaborative situations" [Benford et al. 1995, p. 242].

\subsection{Telepresence Systems}

Fuchs et al. [1994] conceptualized realistic telepresence systems based on a multi-camera ("sea-of-cameras") approach, which was later built on with multiple Kinect cameras [Maimone and Fuchs 2011]. The systems from Beck et al. [2013] and Kulik et al. [2018] enables projection-based multi-user collaborative interaction by tracking the users' behaviors and appearance through multiple RGBdepth cameras. They found similarities in mutual understanding of pointing and tracing gestures for both, local and remote collaborating groups. Billinghurst and Kato [1999] demonstrated that these principles also apply to MR and AR applications . Otsuka [2016] created MMSpace, a system supporting kinetic displays mirroring the remote user's head position in group-to-group conversations, which they find to be superior to a static avatar. Holoportation [Orts-Escolano et al. 2016] allows to project users tracked through multi-camera systems realistically and reliably onto AR devices. In summary, telepresence systems can replicate appearance and behavior with high quality based on RBG/RGB depth sensing. However, two limitations arise: 1 ) the dependability of a clean optical path between camera and user, such as for replicating gaze or facial expressions, which would be hindered by an HMD, and 2) the modification of behavior based on real-time point cloud data is a fairly complex task that, to our best knowledge, has not yet been addressed in the present context.

\subsection{Avatar-Mediated Systems}

Avatar-mediated systems allow for virtual embodiment through avatars [Slater et al. 2010]. Avatars can be defined as virtual characters driven by human movements [Bailenson and Blascovich 2004]. Among early works, Slater, Steed, and colleagues investigated the feasibility and impacts of multi-user avatar-mediated communication [Slater and Steed 2002; Tromp et al. 1998] using the DIVE software for distributed virtual environments [Frécon and Stenius 1998]. The replication of behavior was limited to keyboard input, and participants reported a lack of feedback due to missing body movement and body language [Tromp et al. 1998]. Steptoe, Roberts and colleagues investigated additional modalities for social interaction by including eye gaze [Roberts et al. 2009; Steptoe 


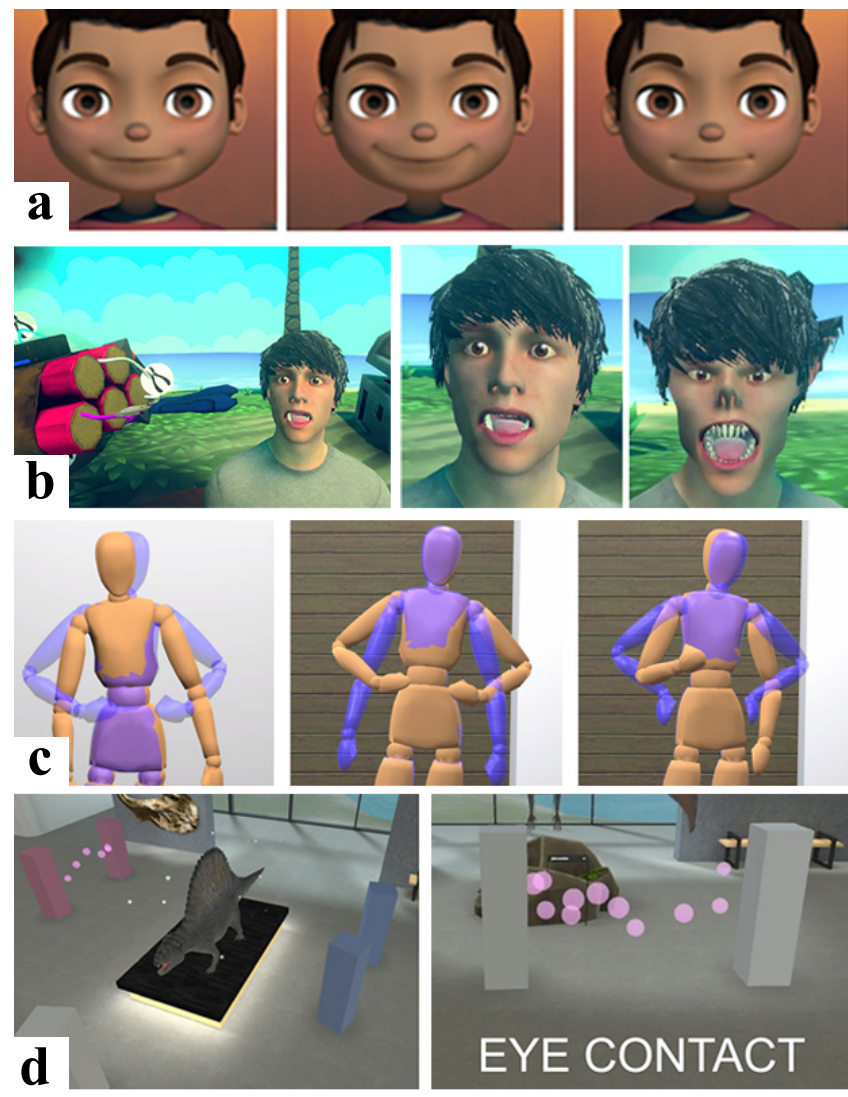

Figure 2: Augmentation examples. a) modified smile behavior (adapted from [Oh et al. 2016] ๑ 2016 Oh, et al.), b) augmented emotions, (adapted from [Hart et al. 2018a], ๑ 2018 Hart, et al.) c) augmented nonverbal mimicry [Roth et al. 2018c] (๑ 2018 Roth, et al.), d) visual transformation, substitution, and amplification of social behaviors [Roth et al. 2018a] (@ 2018 IEEE/Roth, et al.).

et al. 2009]. Bente et al. [2008] included gaze and hand tracking in a desktop-based avatar-mediated communication platform. With improvements in body motion tracking techniques, pose recognition and kinematic solving [Shotton et al. 2013], full-body motion can be replicated to avatars from RGB-depth sensing [Suma et al. 2011] or passive marker systems [Roth et al. 2016b; Spanlang et al. 2014]. Latoschik et al. [2019] investigated scalability and user experience of social VR systems with multiple agents, and found that the perception of fluidity, synchrony and annoyment suffers from higher latencies simulated through multiple clients (2-125), whereas the more agents present in the scene increased the perception of possible interactions and co-presence. Additional sensors allow for a replication of facial expressions and gaze to avatars, for example, using semi-immersive platforms with a Fishtank VR metaphor [Roth et al. 2016c] or an HMD integration [Li et al. 2015]. Piumsomboon et al. [2018] demonstrated the dynamics of avatars as such with respect to avatar size in symmetric and asymmetric remote collaboration in MR with redirected gaze and gestures. Steed et al. [2012] combine both avatar-mediated and telepresence principles to asymmetric communication platforms.
Both telepresence systems and avatar-mediated systems are capable of being displayed via various technologies. Due to the flexibility of the dynamics of avatars, which are, by nature, an exchange medium for human behavior, we based our architecture on an avatar-mediated system. While the realism of both appearance and behavior replication can be seen as reduced in comparison to sophisticated telepresence systems such as holoportation [Orts-Escolano et al. 2016], the systems from Beck et al. [2013] or Maimone and Fuchs [2011], novel approaches to the personalization of avatars by means of photogrammetric scanning can close the gap of reduced appearance realism [Achenbach et al. 2017; Waltemate et al. 2018].

\subsection{Augmentation of Social Behaviors}

Bailenson et al. presented the concept of transformed social interaction which bases on the idea to decouple visual feedback information from actual physical behavior in mediated environments [Bailenson et al. 2004, 2005]. They argued that nonverbal behavior can be transformed strategically (i.e., rendered nonveridically), for example, to direct the attention of a speaker to multiple listeners. They found that the augmentation resulted in greater agreement compared to a non-augmented gaze or a reduced gaze behavior. However, participants in the augmented condition perceived less social presence. Bailenson et al. [2006] further explored mapping behaviors to the appearance and form of a simplified "emotibox" avatar by using facial feature tracking to deform it (e.g., changes of size). The co-presence ratings and emotion transmission were lower in the avatar condition compared to video or voice only. However, there was more verbal and nonverbal disclosure of information in the "emotibox" condition [Bailenson et al. 2006]. Boker et al. [2009] explored the modification of head movements and facial expressions by means of modifying active appearance models in real-time interactions. By doing so, they found that these manipulations in turn had an impact on the behavior of the communication partner.

Oh et al. [2016] modified the facial expressions of avatars in desktop-based dyadic interactions and found that an increased smile increased the positivity of the evaluation of the conversation (Fig. 2 a). Hart et al. recently presented a dynamic appearance modification of avatar faces as a concept for sharing emotions [Hart et al. 2018a,b] (Fig. 2 b). They modify the expression of an avatar based on emotional cues, to apply this augmentation to collaborative video games. Roth et al. [2015] proposed the HAAT concept, which is based on the idea of using the dyad as level of analysis for augmentations, in order to account for bidirectional adaptation and synchronization processes. The underlying system then, for example, reacts to user behavior to augment the communication in order to foster known communication phenomena such as rapport [Gratch et al. 2006; Tickle-Degnen and Rosenthal 1990]. To drive reactive agents, social communication signals of the human users have to be interpreted through social signal processing [Vinciarelli et al. 2009; Wagner et al. 2013] and mapped to agent reactions. HAAT thus takes into account human and technological aspects to actively mediate virtual communication in the social dimension. Roth et al. further explored hybrid and augmented gaze [Roth et al. 2018b], augmented mimicry [Roth et al. 2018c] (Fig. 2 c), as well as visual transformations of social phenomena using HAAT in multiuser VR that lead to increased social presence [Roth et al. 2018a] (Fig. 2 d). 
With regard to the triggering of behaviors and augmentations, Nagendran et al. [2014] present a framework for avatar control. They propose the execution of avatar behavior through microposes executed by the user and tracked by an input device. Aside from scientific explorations, many forms of semi-automatic and automatic social augmentations are present in today's social VR applications and video games [Kolesnichenko et al. 2019]. For example, users can select a facial expression or gesture for their avatar to perform. Similarly, in video games such as Overwatch (Blizzard) or Fortnite (Epic Games), emotes, meaningful gestures such as "thanks", "group up" or dances can be selected and are then executed by the avatars. Those may include additional icon/emoticon visualizations. Moreover, many games today include automatic dialogue exchange between player avatars or between non-player characters and player avatars.

2.4.1 Categorizing Social Augmentations. Fig. 2 depicts some of the above mentioned augmentation examples. Reviewing the presented literature and applications, social augmentations can be categorized based on the following elements:

\section{- The trigger and target modalities}

The behavior modalities used to trigger or display an augmentation (for example, pressing buttons, speech/voice dependence, body motion, proxemics)

- The phenomenological level The phenomenological level the augmentation is executed on or triggered by (for example, a dyadic phenomenon vs. a group phenomenon, conscious vs. non-conscious behaviors)

- The ecosystem of origin

The origin or the world the augmentation is created by (for example, whether the augmentation aims to present natural behavior such as body motion or facial expressions, or artificial affordances such as visual substitutes or amplifications)

\section{- The modification persistence}

Whether or not the augmentation leads to persistent or nonpersistent simulations (for example, augmenting natural behaviors despite the fact that users are presented to themselves by avatars can lead to a decoupling between the simulations of multiple users)

\subsection{Discussion}

Both telepresence systems and avatar-mediated systems have demonstrated the functionality to replicate a high degree of nonverbal behavior [Kulik et al. 2018; Orts-Escolano et al. 2016; Otsuka 2016; Roth et al. 2016c]. To our knowledge, the combined replication of body movement, facial expression, and gaze behavior in a shared and $H M D$-based avatar-mediated system has not yet been presented, and represents a first requirement (RE1) in order to support variable social augmentations features.

With regard to the augmentation of behaviors, previous approaches are limited to linear transformations, such as dampening or amplifying of facial cues [Boker et al. 2009; Oh et al. 2016; Roth et al. 2018c] or modification based on intra-personal information [Bailenson et al. 2006]. Bailenson et al. [2008] discussed the presentation of real-time information of social cues during communication, and Roth et al. [2018b] implemented a speaker-listener based paradigm of gaze augmentation. Some studies have investigated real-time augmentations of body behavior [Roth et al. 2018a,b, 2015] with multiple users based on nonlinear, bidirectional information exchange. However, to the best of our knowledge, no discussion about system architectures and extension to a more generic use and automatic augmentation exists. Such approaches would require a automated status analysis of an interaction (RE2), a social intelligence that makes decisions on the augmentation (RE3) and a sophisticated blending mechanism for the augmentations (RE4). The architecture we propose tackles these requirements. It allows for a high degree of behavior retargeting with the augmentation of nonverbal behaviors based on networked information exchange and can be used to modify different behavior modalities based on centralized decision-making, which we will describe in the following section.

\section{ARCHITECTURE}

\subsection{Components}

Because each user's own behavior should be presented to them in its original form, the network architecture relies on an asynchronous, distributed server-client architecture. While exchange data are synchronized between client machines, the simulations can have asynchronous states with regard to their visual rendering.

The architecture is based on three main components: i) a status analysis component (SAC) to analyze the current status of the communication, ii) a social intelligence and modification component (SIMC) to decide upon augmentation and transformation of behaviors, and iii) a behavioral blending component (BBC) to adequately blend the respective modalities and to provide decoupled visual feedback (see Fig. 3).

The SAC analyzes the intra-modality status of the individual as well as the inter-modality status of the communication for both users. To assess the communication status, such as the level of synchronicity [Hove and Risen 2009], agreement or rapport [TickleDegnen and Rosenthal 1990], the SAC gathers data from the sensing layer of both clients locally, analyzes the data of the individual interactant or both interactants, and judges the current status. For example, in the case of the current prototype, the conversation can be analyzed on the basis of speaker/listener status or the level of agreement based on head nod frequencies; see also Sec. 4.2.1. The status, respectively triggers are then passed on the SIMC.

The SIMC acts upon the status information. Based on the information from the SAC, it decides upon necessary transformation, passing through adequate behaviors in the case of functional (i.e, regular and adequate) communication or transforming behavior in the case of dysfunctional (e.g., disturbed by transmission or user) nonverbal communication. The decisions regarding which behaviors to augment and to what degree are made by the SIMC. In the current protoype, the SIMC is implemented in static form based on a state machine (see Fig. 6). For example, humans typically focus on the speaker and pay attention while listening and do not tend to mimic semantic gestures made by the speaker, such as pointing or beat gestures. Thus, it may be inadequate to augment the mimicking of complex gestural behaviors while listening. Such contexts needs to be taken into consideration (see also Sec. 4.3.1).

In the BBC, blending from the exchange layer to the augmentation layer and vice versa are processed, and the result serves as 
a) Architecture

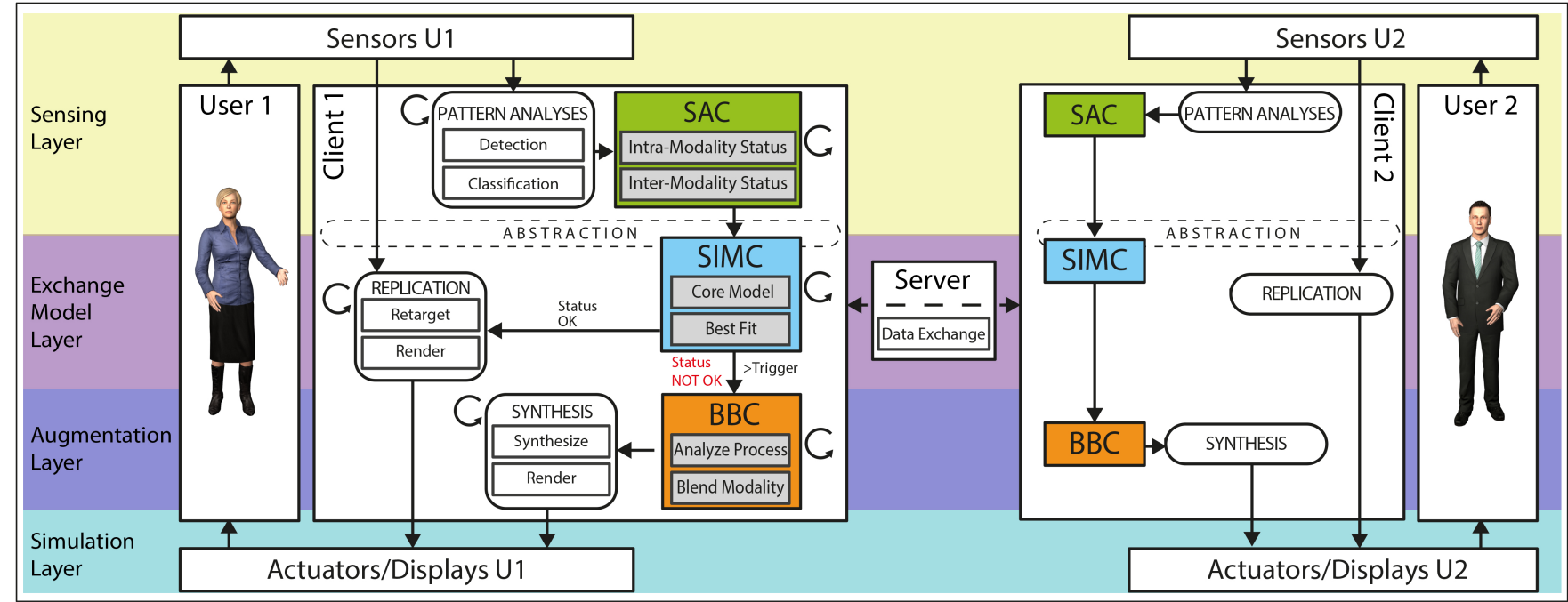

b) Augmentation Example (Gaze Focus)

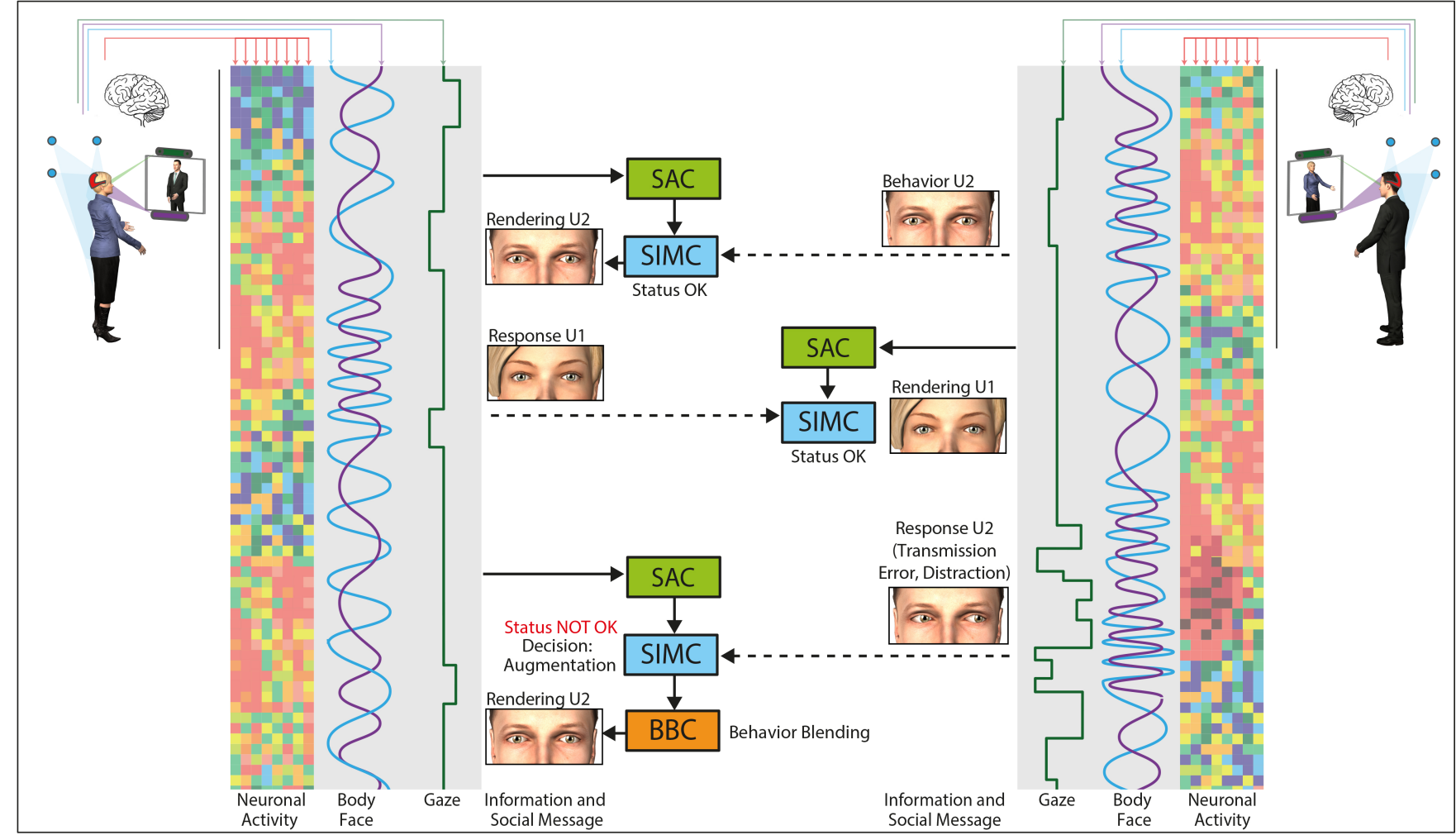

Figure 3: a) Proposed architecture. Each local simulation is responsible for detecting the status and trigger features, retargeting/replicating the behaviors, and synthesizing augmentations. Network synchronization is achieved through the exchange model layer. The status analysis component (SAC) in each simulation analyzes intra- and inter-modality status of communicative patterns, and forwards the information to the social intelligence and modification component (SIMC), which decides upon an appropriate modification. The behavioral blending component (BBC) reacts to forwarded decisions and blends modalities between the augmentation layer and the exchange model layer. b) Exemplary intervention principle. Based upon multimodal behavior data input, the SAC informs the SIMC, which decides that gaze behavior has to be augmented (e.g., due to a transmission error). The BBC blends augmented behaviors and alters the social message exchange, for example, the attentional focus. The integration of neuronal data is subject to present work [Roth et al. 2019]. 


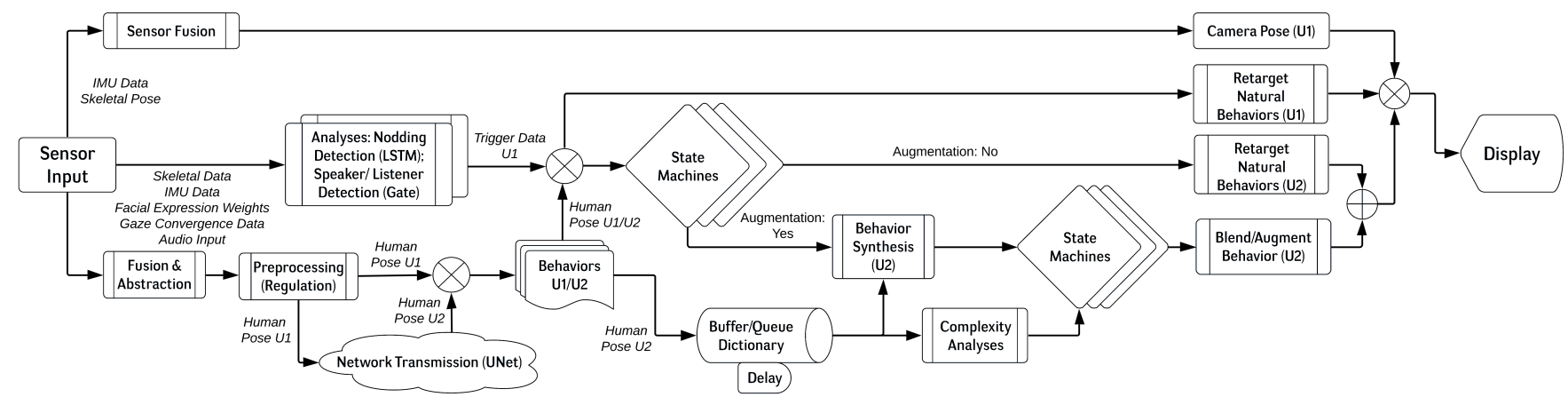

Figure 4: Simplified data flow of the implementation of the prototype.

output for the simulation layer and rendering. The blending, and thus the visualization of augmentations, depends on timing. First, the $\mathrm{BBC}$ decides on the appropriate delay between the decision for the augmentation and the actual execution. Second, in the cases of artificial mimicry or social gaze patterns, the timing and form of blending is of importance. Whereas eye movements are rapid movements, moving an arm or performing a body sway are typically slower movements, and thus the complexity of the movement and the distance between the starting and ending points need to be taken into account. Similarly, the timing and presentation of more abstract visual augmentations (e.g., emoticons, smileys, highlights, and the like) would be controlled by the BBC.

\subsection{Data Layers}

With regard to the underlying data transportation and processing, the architecture relies on four abstract layers (see Fig. 3 a):

- 0 - Sensing Layer. The raw sensor data received by the simulation via sensor APIs and third-party integrations. Sensing layer data are not synchronized between the clients.

- 1 - Exchange Model Layer. Interpreted raw data are mapped onto a template pose (kinematic solver) model used to access, distribute, and synchronize abstracted behavioral data between server and clients. This human pose model is based on high-level interpretation of the behaviors (joint angle representations) and blendshape weights. The model allows for compatibility with different avatar types.

- 2 - Augmentation Layer. The augmentation layer transmits transformed behavioral data received from the exchange model layer and modifies it in continuous (e.g., body and facial mimicry) or event-triggered (e.g., joint attention or speaker-listener occurrences) form. Data are individual for each client, and not synchronized via the network.

- 3 - Simulation Layer. Controlled by the underlying AI, the simulation layer receives data from both the exchange model layer and the augmentation layer to render the local simulation.

This abstraction allows local access to original behavior data as well as synthesized data for any given point in time.

\section{PROTOTYPE: INJECTX}

InjectX is built in Unity3D (2018.3.14) in combination with Python (3.6) and TensorFlow (1.12) for pattern recognition. A simplified flow diagram is provided in Fig. 4. Based on the Unity3D network API (UNet) and a client-server architecture, injectX is implemented to consider any number of users. In the current study, we did assess and construct the system for two local users, but it was based on the underlying distributed system architecture presented in Sec. 3. Input from behavior tracking is prepocessed, applied to a user's selfrepresentation through the exchange model layer, and continuously synchronized to peers via UDP: one's own updated data are sent to a server instance of the application at regular intervals, and the central server synchronizes all data with all clients.

A server can run either as dedicated instance or run its own client as host. Because of the non-competitive nature of the simulation, clients have complete authority (i.e., local authority) to edit their local player's data. The simulation is rendered to FOVE 0 HMDs (FOVE, Inc., 2560x1440 px, 100 degree field of view, $70 \mathrm{~Hz}$ refresh rate). We fused the FOVE 0 inertial measurement unit data with positional tracking of the motion tracking system. Furthermore, we applied drift correction (linear interpolation) whenever absolute rotation and relative rotation difference exceeded a threshold (1 degree).

\subsection{Behavior Tracking and Retargeting}

We based our integration of body motion on previous works [Roth et al. 2016b,c; Spanlang et al. 2014]. We used an OptiTrack Flex 3 system (18 cameras, $120 \mathrm{~Hz}, 640 \times 480$ px) [OptiTrack, Inc. 2018] and a marker set with 37 reflective markers (see Fig. 1 a,b). An estimated skeleton pose is retreived from NaturalPoint, Inc.'s Motive application. The Unity integration receives position and orientation of the skeleton's root joint (hip) and the orientation of the child nodes (sensing layer). To synchronize body motions between simulations in the exchange model layer, we construct a human pose from the skeleton data. We infer into Unity's human pose solver late in the game loop to overwrite the internal center of mass estimation of the hip (root node) with original data, which reduces artifacts such as foot skating.

We use BinaryVR Inc.'s facial expression sensing development kit to gather information about lower facial deformation. Therefore, 


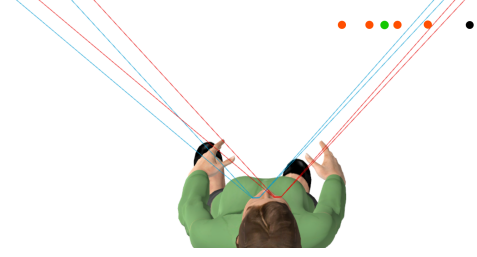

(a)

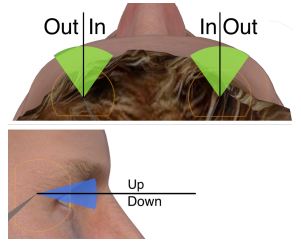

(b)

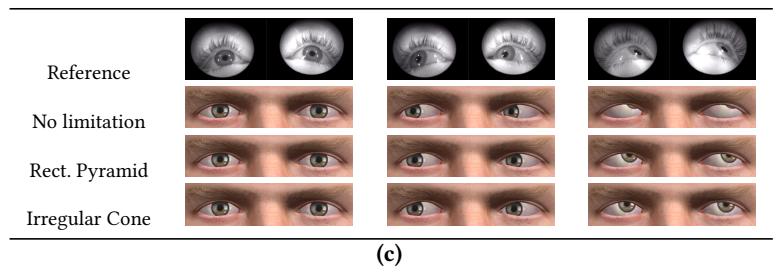

Figure 5: Gaze preprocessing. (a) Samples outside (black) the frustum (blue/red) are dampened. Valid points (yellow) are smoothed (green). (b) Angle limits define legal rotations. (c) Regulation method comparison. We use the irregular cone.

a 3D depth sensing device (Pico Flexx) that uses infrared illumination is affixed to the HMD (see Fig. 1 a). The 2D and depth images are used by BinaryVR, Inc.'s software to generate facial expression deformation parameters by tracking landmark locations [Yu and Park 2017]. Tracked expression parameters in the form of blendshapes include, for example, jaw-open, puff, and smile. Similar to [Roth et al. 2016c], we retargeted the input data (sensing layer) to the facial blendshapes (exchange model layer), using weighted look-up tables (LUTs). The LUT settings can be stored to allow for the mapping of virtual characters from multiple sources, given that the characters provide blendshapes for animation.

We paid special attention to the inclusion of eye gaze behavior in the current prototype. Eyesight direction is detected by using the FOVE 0's binocular eye-tracking system $(120 \mathrm{~Hz})$, which captures the reflection image on a hot mirror showing infrared light emitted toward a user's cornea. Combining the two individual eye's vectors, the FOVE integration calculates the intersection point to estimate the convergence point in the virtual scene, meaning the location the user is gazing at [Wilson and Seko 7 09].

While the FOVE 0 provides relatively robust tracking, tracking dropouts (invalid data) and the requirement of retargeting the data to avatars called for additional preprocessing (depicted in Fig. 5): (1) Convergence points are considered valid if they are inside one or both of the HMD's virtual camera frustums. (2) We smooth the data points with an unweighted moving average filter with a window size of four samples [Špakov 2012], adding a three-sample delay. (3) We rotate the digital representation's eyes toward the smoothed convergence point. (4) Local eye rotations outside their natural range of motion, defined by an irregularly shaped cone [Aristidou and Lasenby 2011], are replaced with the most similar rotation within their specified range of motion. As a result, illegal rotations are replaced with natural equivalents that best match the target rotation while not looking eerie, with pupils hidden behind eyelids. An outcome comparison of evaluated approaches is shown in Fig. 5 c. We integrated artificial blinks to increase the humanness by using blendshape retargeting. Blinks are synthesized and not derived from the sensor data. Blinks appear randomly (uniform distribution), with a frequency between $f_{\min }=2 \mathrm{~Hz}$ and $f_{\max }=10 \mathrm{~Hz}$, and for a random duration between $100 \mathrm{~ms}$ and $150 \mathrm{~ms}$, approximated from previous findings [Liversedge et al. 2011].

In our current prototype, voice signals are captured using unidirectional microphones suspended from the tracking area scaffold. Audio input is integrated with Placeholder Software's Unity plugin Dissonance Voice Chat and could be transmitted (voice over IP) via distinct network channels [Unity Technologies, ApS 2018]. injectX focuses on voice (audio input) as augmentation trigger in the current state. Any microphone/speaker combination could be used for remote audio transmission.

\subsection{Status Analysis and Monitoring}

4.2.1 Detection and Classification of Communicative Patterns. As a prototypical signal for the interpretation of dynamic phenomena by the SAC, we used supervised learning based on a long short-term memory (LSTM) network to detect head nods as one indicator for agreement. Four modules were included in order to first train the network and later allow for a real-time classification: 1) a standalone classification application (Python, TensorFlow), 2) a replay system to record and play back interactions (Unity), 3) an annotation system that allows quick annotation of playbacks to use as training data (Unity), and 4) a trigger module that communicates with the classification application through a socket connection and informs the main application of detected states.

For the prototype, we used a static recurrent neural network (RNN) with an LSTM cell (hidden units: 100; time steps: 50). The LSTM was trained on 1,458 samples (head nods: 729; batch size: 130; epochs: 200; learning rate: 0.001 ; optimizer: adaptive moment estimation) of three participants' head motion data from the exchange model layer. The network achieved an accuracy of about $85 \%$ in our validation set. For the real-time status analysis, the detections of nodding are passed as functionality of the SAC. Although the functional prototype of the module is limited to head nodding, the server can handle any data input that matches the data-conventions.

4.2.2 Speaker and Listener Detection. The detection of speaker and listener status is based on approaches of previous works [Lee et al. 2002a; Roth et al. 2018b]. We use the audio input to assess the amplitude of the audio input. An audio gate is applied to filter any noise over a threshold. Speaker status is then passed on to the SIMC as functionality of the SAC.

\subsection{Augmentation Features}

4.3.1 Mimicking of Behaviors. Nonverbal mimicry can be described as the motor imitation of another's behavior and is associated with positive impacts such as affinity, liking, and rapport [Lakin et al. 2003]. To induce artificial mimicry, we based the implementation on the approach from Roth et al. [2018c]. While [Roth et al. 2018c] used a naïve periodic induction of mimicry, the present prototype stresses the decision-making process of the underlying SIMC component, which makes decisions on the basis of the adequacy of a manipulation. Fig. 6 shows a state machine deciding upon an augmentation. We analyze the speaker and listener statuses as well as the complexity of the queued mimicry behavior (of the interaction partner). 


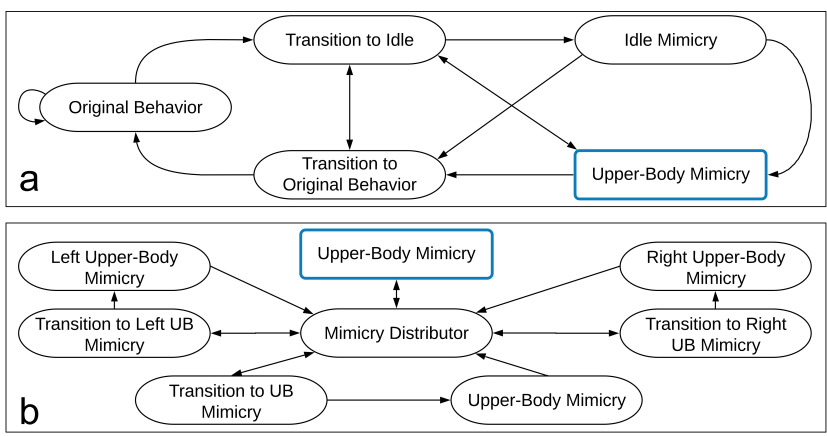

Figure 6: Higher and lower level decision-making. a) Main states. Mimicry is augmented if a listener is detected. b) Secondary ruling system. The mimicry distributor decides upon an augmentation, depending on the (buffered) speaker's motion complexity. If left arm movements are complex, the left arm is excluded from the augmentation.

To assess the motion complexity, we average the hand position distances between two frames over a $0.35 \mathrm{~s}$ window in the buffered values using a coroutine $(60 \mathrm{~Hz})$. Critical (complex) motions are identified by a threshold ( 0.0025 Unity values). The SIMC makes a decision regarding a mimicry augmentation scanning a $2.5 \mathrm{~s}$ window for critical values. The decision can be made individually for each arm. This approach can be generalized, for example, to support facial mimicry.

4.3.2 Attentive and Averted Gaze. Similar to the work from Roth et al. [2018b] the implemented gaze model for evoking attentive gaze is based on statistical distribution of saccadic movement and fixations [Lee et al. 2002a]. It reacts to trigger events in the form of invoking directed gaze (see Fig. 1 e,f).

We improved the previous approach by limiting the radial extension of gaze shifts. Averted gaze is synthesized by picking a random direction from a straight circular cone spread around the direction vector from the interlocutor's eye center to the previous eye convergence position. As human saccades mostly occur with a magnitude of 15 degrees or less [Bahill et al. 1975], the random direction maximally deviates by 15 degrees from the previous direction. Transitions of gaze behavior between the exchange model layer and the augmentation layer are performed using a cubic Bézier curve. Both attentive gaze and the artificial injection of mimicry are based on state machines implemented in Unity.

\subsection{Behavior Blending}

While gaze and facial movements are relatively rapid motions that suffer little from imperfect functions of interpolation, the blending of body movement required further investigation due to the higher mass and slower pace of motion. We therefore evaluated typical blending functions such as linear and spline interpolations (see Fig. 7). While Rose et al. [1996] reported a duration of $0.3 \mathrm{~s}$ to 0.6 $\mathrm{s}$ for motion transitions, Lee et al. [2002b] as well as Kovar et al. [2008] used $1 \mathrm{~s}$ to $2 \mathrm{~s}$, and Kovar et al. [2008] used a duration of $0.33 \mathrm{~s}$ for motion blending. To find an adequate blending functions for body motion, we conducted a user study $(N=36,22$ females,

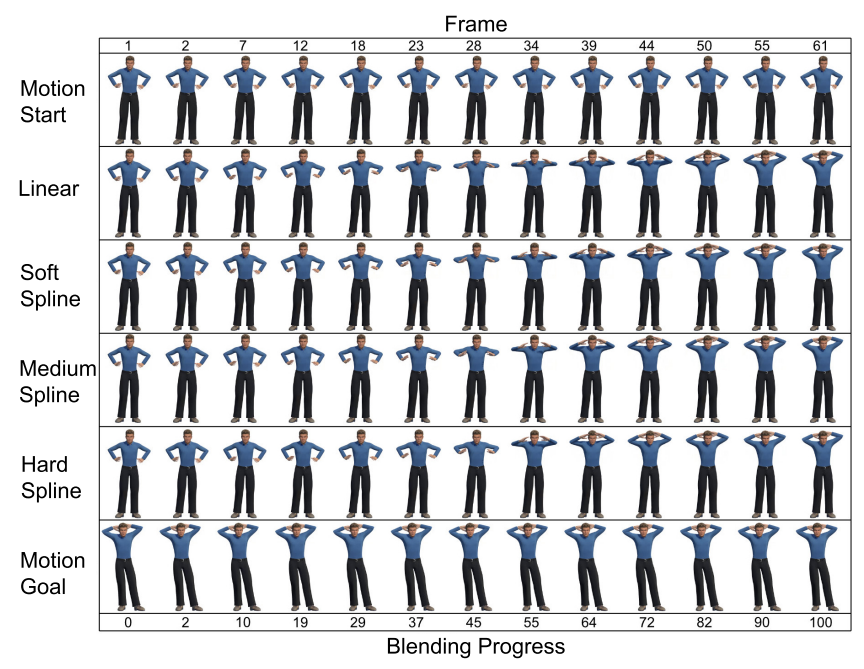

Figure 7: Blending functions used for initial tests.

\section{Listing 1: Simplified Example: Adaptive Motion Blending}

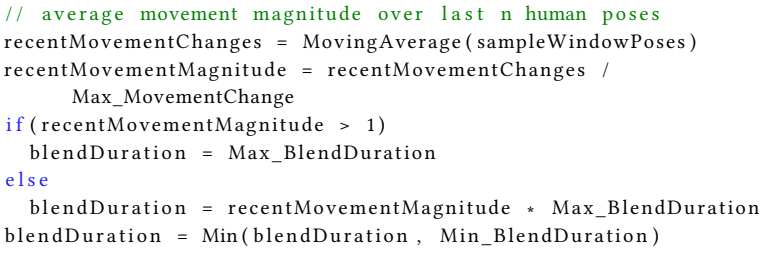

$\left.M_{\text {age }}=21.58\right)$ evaluating three combinations of short motions (M1: slow idle movement a vs. slow idle movement b, M2: slow idle a vs. rapid movement a, M3: rapid movement a vs. rapid movement $b$ ), evaluating four blending types (linear, soft spline, medium spline, hard spline) and three different blending durations (500 ms, 1000 $\mathrm{ms}, 1500 \mathrm{~ms}$ ). The animations were pre-recorded and blended using a playback module that was implemented to annotate behavior samples and evaluate different features. Participants evaluated the resulting 36 rendered videos with regard to naturalness (artificial vs. natural), animacy (mechanical vs. organic), affect (positive vs. negative), and eeriness (eerie vs. reassuring) derived from [Ho and MacDorman 2010] on 11-point semantic differentials.

Analysis of variance revealed that the motion type (i.e. the kind of motions that were combined) significantly affected the ratings on all four measurements $\left(p^{\prime} s<=.008\right)$, whereas no other significant main effects were found. There was no clear image on an optimal function from our results. However, on average, soft- and mediumspline blending with $1000 \mathrm{~ms}$ duration had overall good ratings in being perceived natural, organic, reassuring, and positive.

We further chose to develop an adaptive blending mechanism for body motion that is based on the distance between all joints that need to be blended. The blending is based on a soft spline, and the blending duration is adjusted depending on the motion magnitude of recent movements. The approach is depicted in Listing 1. 


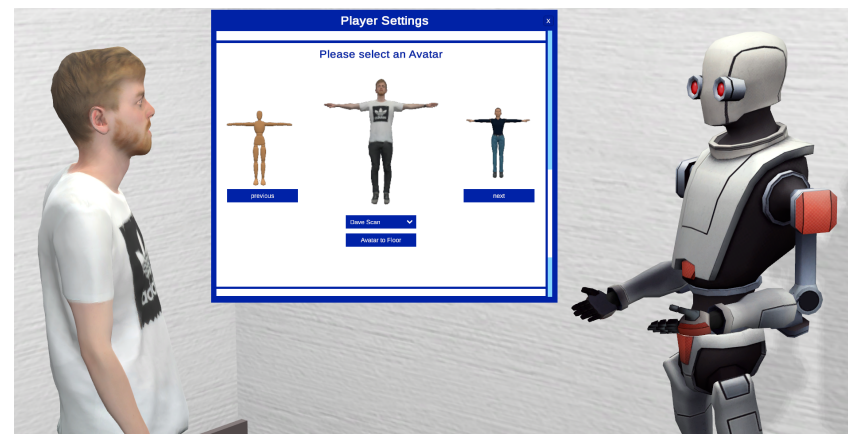

Figure 8: A character integrator and selector allows to integrate and load fbx specified rigged characters.

\subsection{Character Integration}

injectX is built for humanoid avatars and can utilize characters that fulfill the requirements for the humanoid specification in Unity, which requires a minimum of 15 bones [Technologies 2018]. This allows a large variety of characters from different platforms, including personalized scans, in FBX format. Characters can be selected through a graphical interface (see Fig. 8). Rationally, the retargeting of behavior is limited to the features of the respective character. Characters can be scaled through parametrization, and floor contact can be calibrated through a function that takes the lowest mesh point and accounts for the difference.

With our approach to the character integration, it is also possible to integrate avatars that are generated through photogrammetric scanning [Achenbach et al. 2017; Latoschik et al. 2017] (see Fig. 4.5 left). This, for example, reduces artifacts such as internal mesh collisions that are typically present when avatars have different body measures than their users, for example, when poses such as those displayed in Fig. 7 are performed.

\subsection{System Latency}

We tested on two machines (Intel Core i7-8700K, 3.70 Ghz, GeForce GTX 1080 Ti, 16 GB RAM, Intel I219V network), interconnected with a Gigabit switch (Cisco Catalyst 365048 2X). For their tracking and rendering, FOVE reports an approximate of $20-30 \mathrm{~ms}$. The motion-to-simulation latency of the facial expression replication, body movement replication, and gaze replication were estimated by frame counting $(120 \mathrm{~Hz}$ video camera, Acer H6517ST projector with a known input lag of $M=34 \mathrm{~ms}$ ) to $M=144.62 \mathrm{~ms}$ (facial expressions), $M=113.04 \mathrm{~ms}$ (body motion), and $M=337.0 \mathrm{~ms}$ (gaze replication). The network transmission accounted for $M=$ $10.89 \mathrm{~ms}$. The augmentation and blending adds a simulation delay according to the queue times parameterized and respective function applied. There were no noticeable delays between real and modified behaviors when excluding these queue times.

\subsection{Reliability}

InjectX supports the replication of body movement, facial expressions, and gaze that are only limited by the sensor capabilities (RE1). We performed further steps to improve the quality, such as the preprocessing of gaze data, character scaling, as well as a variable LUT component for facial expressions in order to address character blendshape variability.

Regarding the trigger detection, the prototype supports a rather robust detection of the speaker-listener status through the presence of voice, while the ML based detection requires an improvement in both, method and reliability. Regarding the blending reliability, the prototype supports reliable gaze and body motion blending. The latter is optimized using a magnitude adaptation for the blending time (see Listing 1). For body motion, pose conflicts can occur with strong divergence between the physical measures of both users and avatars, which is a problem not targeted in the current prototype and subject to future work.

\subsection{Scalability}

The system is, in theory, scalable to any number of users due to the client-server model. We specifically considered this when designing the abstraction of the data layers. Each client has authority over simulated behavior and is therefore not dependent on a reliable protocol that ensures the arrival of packets. The asymmetry of the architecture distributes the workload to the clients limited only by transmission bandwidth. Due to the abstraction of behavioral data through the exchange model layer (human pose model), the architecture is not dependent on specific sensory input and may utilize any tracking type for body motion that delivers skeleton data/inverse kinematic retargeting [Roth et al. 2016a], any facial tracker that supports blendshape data, and any eye tracker that delivers gaze target data.

Most importantly, the underlying components and features for the augmentation are scalable to other behavior and augmentation types and support multi-layer triggers and augmentation modalities. For example, facial mimicry could be performed by the same principles. The status analysis and social intelligence can be extended by additional rules or triggers.

\subsection{Comparison to Previous Work}

The approach by Piumsomboon et al. [2018] is an avatar-based system comparable to ours. While they developed Mini-Me for mixed-reality applications, we tested solely VR simulations. In comparison to their approach, we include both natural and synthesized gaze, whereas their system relies on artificial gaze based on the method from Borland et al. [2013]. While the approach by Roth et al. [2016c] allows transmission of facial expressions, gaze, and body motion, it is limited to a semi-immersive projection setting and does not allow for avatar embodiment.

With regard to the augmentations, we extend previous works on transformed interactions [Bailenson et al. 2004, 2008, 2006] by including reactive transformed behavior [Gratch et al. 2006] that is based on communication patterns as the level of analysis. Compared to previous approaches for social augmentations [Roth et al. 2018 b,c] that were limited to a single nonverbal channel, the presented architecture allows for different augmentations in a general fashion. Furthermore, our decision-making is more sophisticated compared to previous studies, and we have presented, to the best of our knowledge, the first architecture that considers bidirectional augmentations in multi-user immersive environments that not only affect both users but also depend on the behaviors of both users. 


\section{DISCUSSION}

We have proposed an architecture for simulations that supports social augmentations. Based on the data layer abstraction and the three presented components, the architecture can handle the multilayered behaviors necessary for social augmentation systems. The abstracted data layers allow for decoupling sensing and data exchange from logical, capsuled components.

Regarding the realistic replication of behaviors (RE1), our prototype supports a high degree of behavior replication, and future sensor technologies can be included to improve this quality. While some telepresence systems (e.g., [Orts-Escolano et al. 2016]) may be superior in appearance realism, they do not allow for HMD-based rendering. Based on the architecture proposal, our prototype supports the automated status analysis of an interaction (RE2) in the form of agreement analysis (head nodding) and speaker/listener detection. The system provides a social intelligence that supports decision-making (RE3) on the basis of state machines that decide upon an augmentation based on the trigger signals and communication status, taking motion complexity information into account. Finally, we provided a blending mechanism (RE4) that can blend gaze behavior and body motion on the basis of continuous monitoring, time frame constraints, and adaptive motion blending. Some limitations are inevitable.

\subsection{Limitations}

First, gaze augmentation is only practicable for situations in which communcation partners face each other as we do not yet kinematically solve the upper body and head motions required if an averted listener is facing in a complete opposed direction. Second, we discovered occasional blending conflicts in body motion and gaze, which may be solved by further filtering. These were not as severe, however, as the internal mesh collisions we observed when the physical body of a user had different measurements (e.g., circumference of the belly). Rationally, these are substantially reduced with personalized avatars (i.e., measure adjusted/photogrammetry scanned) avatars. While a further elaboration of this problem was not the subject of the present work, future developments should consider adjusting augmented motions based on the users' measures to avoid such conflicts, for example through physically based models that account for mesh collisions in addition to the human pose solving that is used in our prototype. Third, the gaze latency is considerably high. Further investigations need to be conducted on how the latency of behaviors affect and disturb the communicative patterns between two users. Fourth, the SAC in the present architecture analyzes the true (real, physical) motion, irrespective of the augmented motion. This factor needs to be further investigated and is also a point of further discussion and critique. For example, in the case of mimicry, if user 1 mimics the already augmented behavior of user 2, communication patterns might diverge. Therefore, it is also a question of the time span and amplitude of an intervention that has to be further explored. We have not yet found a solution for this problem. One solution may be to present the augmentation status to the users. Another approach could be to also synchronize the augmentation layer and exclude simultaneous augmentations. Fifth, we did not (yet) include mechanisms for users to stop or prohibit augmentations. The latter will be discussed in more detail in Sec.
5.3. Sixth, the system is limited to the presented sensing. We did not include finger tracking in the current prototype, and thus the hands are displayed in a fixed (relaxed) pose.

\subsection{Lessons Learned}

One of the most helpful features in exploring social augmentations with injectX was the implementation of a recording and playback module. This module was essential throughout development as synchronous logging of parameters was a key test environment and this allowed for more exploration of augmentations. Another essential step of progress was the decision to regain control over pose retargeting and infer with the retargeting system that Unity provides, meaning overwriting the retargeting and correct for the original hip positioning in the latest function of the game loop (lateUpdate()) to correct for solver mismatches. With the prototype, we had the objective of exploring multimodal augmentations. However, literature to that regard gives provides insight into the timeliness of phenomena such as mimicry, which is broadly understood but not at all parameterized by the literature.

\subsection{Ethical and Societal Considerations}

Without any doubt, the modification of behaviors by an artificially driven intelligence can weaken human autonomy and information control. For example, we do not know what types of unintended results can arise from augmented behaviors and how to best make users aware of actual augmentations. It is, therefore, important to discuss potential risks such as data security, privacy, transparency, and restricted autonomy. While it may be appropriate for experimental research projects and lab studies to retain control over collected data and any modifications thereto, the rules have to be rethought in terms of how much control is given to AI to retrieve individual data and construct artificial models of behavior in real time. The application of models of "standard" human behavior requires characterizing this behavior in larger populations, taking potentially influencing factors (e.g., sex, age, culture) into account. We considered different aspects of such simulations and propose countermeasures that could mitigate the risks evolving from such systems with regard to privacy and data security:

- Introduction of a digital identity to protect social AI data

- Ensure transmission protocol security

Furthermore, future human-machine interfaces for virtual simulations will take a shortcut and avoid the detection of actual physical behaviors and will, instead, foresee behaviors based on neurophysiological signals [Lecuyer et al. 2008; Slater 2014]. At that point, the machines will literally know more about us than we do. It must therefore be made transparent to what degree and based on which information sources social AIs are trained (behavior, neurophysiological correlates). The following countermeasures for mitigating the risks of decreased autonomy could be enforced:

- Constantly present the status of interference

- Constantly allow users to stop any process of interference

- Constantly inform users about data tracking and storage 


\section{CONCLUSION}

This paper presented a novel architecture for the development of systems that support social augmentation. We described the architecture for a distributed system and a prototype that significantly extends comparable approaches that are based on avatar-mediated systems. The prototype is currently capable of replicating body movement, gaze, and facial expressions in an immersive, embodied simulation, as well as performing different augmentations based on sophisticated status analysis, decision-making, and motion blending by an underlying social artificial intelligence. Previous approaches merely focused on the linear transformation of behaviors, while our approach takes into account bidirectional interpretations of communication signals to augment social behaviors in real time based on multimodal input.

\section{FUTURE WORK}

As depicted in Fig. 3, one direction of future work is to feed neural data input into our prototype [Roth et al. 2019], and a next step is to derive status and communicative signals from a wide range of (neuro)physiological data. We also aim at a stronger focus on dyadic pattern analysis to strengthen the status analysis component and assess the status on time-continuous levels, such as a synchronicity measure. Furthermore, we want to apply our body mimicry to facial mimicry scenarios. In addition, we aim at collecting more data to allow for more sophisticated machine learning approaches to detect triggers, construct and blend behavior, and base it on a multimodal analysis rather than the analysis of single modalities. Last, we aim at integrating abstract and transformed visual information of communicative behavior (such as emotes/emoticons, context changes, form and appearance changes) to provide alternative feedback affordances. To support reproducibility, the source code of injectX is available upon request for non-commercial non-dual-use research purposes, given the respective acquisition of third-party licenses.

\section{ACKNOWLEDGMENTS}

We thank Christopher Göttfert and Jonathan Stapf for support.

\section{REFERENCES}

Jascha Achenbach, Thomas Waltemate, Marc Erich Latoschik, and Mario Botsch. 2017 Fast Generation of Realistic Virtual Humans. In Proceedings of the 23rd ACM Symposium on Virtual Reality Software and Technology (VRST '17). ACM, New York, NY, USA, Article 12, 10 pages. https://doi.org/10.1145/3139131.3139154

Andreas Aristidou and Joan Lasenby. 2011. FABRIK: A fast, iterative solver for the Inverse Kinematics problem. Graphical Models 73, 5 (2011), 243 - 260. https: //doi.org/10.1016/j.gmod.2011.05.003

A Terry Bahill, Deborah Adler, and Lawrence Stark. 1975. Most naturally occurring human saccades have magnitudes of 15 degrees or less. Investigative Ophthalmology \& Visual Science 14, 6 (1975), 468-469.

Jeremy N. Bailenson, Andrew C. Beall, Jack Loomis, Jim Blascovich, and Matthew Turk 2004. Transformed social interaction: Decoupling representation from behavior and form in collaborative virtual environments. Presence 13, 4 (2004), 428-441.

Jeremy N. Bailenson, Andrew C. Beall, Jack Loomis, Jim Blascovich, and Matthew Turk. 2005. Transformed social interaction, augmented gaze, and social influence in immersive virtual environments. Human communication research 31, 4 (2005), 511-537. https://doi.org/10.1111/j.1468-2958.2005.tb00881.x

Jeremy N Bailenson and Jim Blascovich. 2004. Avatars. In Encyclopedia of humancomputer interaction. Berkshire Publishing Group.

Jeremy N. Bailenson, Nick Yee, Jim Blascovich, and Rosanna E. Guadagno. 2008. Trans formed social interaction in mediated interpersonal communication. Mediated interpersonal communication 6 (2008), 77-99.

Jeremy N. Bailenson, Nick Yee, Dan Merget, and Ralph Schroeder. 2006. The effect of behavioral realism and form realism of real-time avatar faces verbal disclosure, nonverbal disclosure, emotion recognition, and copresence in dyadic interaction. Presence: Teleoperators and Virtual Environments 15, 4 (2006), 359-372.

Stephan Beck, Andre Kunert, Alexander Kulik, and Bernd Froehlich. 2013. Immersive group-to-group telepresence. IEEE Transactions on Visualization and Computer Graphics 19, 4 (2013), 616-625.

Steve Benford, John Bowers, Lennart E. Fahlén, Chris Greenhalgh, and Dave Snowdon. 1995. User Embodiment in Collaborative Virtual Environments. In Proceedings of the SIGCHI Conference on Human Factors in Computing Systems (CHI '95). ACM Press/Addison-Wesley Publishing Co., New York, NY, USA, 242-249. https://doi. org $/ 10.1145 / 223904.223935$

Gary Bente, Sabine Rüggenberg, Nicole C. Krämer, and Felix Eschenburg. 2008. AvatarMediated Networking: Increasing Social Presence and Interpersonal Trust in NetBased Collaborations. Human Communication Research 34, 2 (April 2008), 287-318. https://doi.org/10.1111/j.1468-2958.2008.00322.x

Mark Billinghurst and Hirokazu Kato. 1999. Collaborative mixed reality. In Proceedings of the First International Symposium on Mixed Reality. 261-284.

Chuck Blanchard, Scott Burgess, Young Harvill, Jaron Lanier, Ann Lasko, Mark Oberman, and Mike Teitel. 1990. Reality Built for Two: A Virtual Reality Tool. Vol. 24. ACM, New York, NY, USA. 35-36 pages. https://doi.org/10.1145/91394.91409

Steven M. Boker, Jeffrey F. Cohn, Barry-John Theobald, Iain Matthews, Timothy R. Brick, and Jeffrey R. Spies. 2009. Effects of Damping Head Movement and Facial Expression in Dyadic Conversation Using Real-time Facial Expression Tracking and Synthesized Avatars. Philosophical Transactions of the Royal Society B: Biological Sciences 364, 1535 (2009), 3485-3495. https://doi.org/10.1098/rstb.2009.0152

David Borland, Tabitha Peck, and Mel Slater. 2013. An Evaluation of Self-Avatar Eye Movement for Virtual Embodiment. IEEE Transactions on Visualization and Computer Graphics 19, 4 (2013), 591-596. https://doi.org/10.1109/TVCG.2013.24

Joseph N Cappella. 1981. Mutual influence in expressive behavior: Adult-adult and infant-adult dyadic interaction. Psychological bulletin 89, 1 (1981), 101.

Emmanuel Frécon and Mårten Stenius. 1998. DIVE: A scaleable network architecture for distributed virtual environments. Distributed Systems Engineering 5, 3 (1998), 91.

Henry Fuchs, Gary Bishop, Kevin Arthur, Leonard McMillan, Ruzena Bajcsy, Sang Lee, Hany Farid, and Takeo Kanade. 1994. Virtual space teleconferencing using a sea of cameras. In Proc. First International Conference on Medical Robotics and Computer Assisted Surgery, Vol. 26.

Michael Gerhard, David J. Moore, and Dave J. Hobbs. 2001. Continuous presence in collaborative virtual environments: Towards a hybrid avatar-agent model for user representation. In Intl. Workshop Intelligent Virtual Agents. Springer, 137-155.

Jonathan Gratch, Anna Okhmatovskaia, Francois Lamothe, Stacy Marsella, Mathieu Morales, R. J. van der Werf, and Louis-Philippe Morency. 2006. Virtual Rapport. In Intelligent Virtual Agents, Jonathan Gratch, Michael Young, Ruth Aylett, Daniel Ballin, and Patrick Olivier (Eds.). Springer Berlin Heidelberg, Berlin, Heidelberg, $14-27$.

Jonathon D. Hart, Thammathip Piumsomboon, Louise Lawrence, Gun A. Lee, Ross T. Smith, and Mark Billinghurst. 2018a. Emotion Sharing and Augmentation in Cooperative Virtual Reality Games. In Proceedings of the 2018 Annual Symposium on Computer-Human Interaction in Play Companion Extended Abstracts (CHI PLAY '18 Extended Abstracts). ACM, New York, NY, USA, 453-460. https://doi.org/10. $1145 / 3270316.3271543$

Jonathon D Hart, Thammathip Piumsomboon, Gun Lee, and Mark Billinghurst. 2018b. Sharing and Augmenting Emotion in Collaborative Mixed Reality. In 2018 IEEE International Symposium on Mixed and Augmented Reality Adjunct (ISMAR-Adjunct). IEEE, 212-213.

Chin-Chang Ho and Karl F. MacDorman. 2010. Revisiting the uncanny valley theory: Developing and validating an alternative to the Godspeed indices. Computers in Human Behavior 26, 6 (2010), 1508-1518.

Jim Hollan and Scott Stornetta. 1992. Beyond Being There. In Proceedings of the SIGCHI Conference on Human Factors in Computing Systems (CHI '92). ACM, New York, NY, USA, 119-125. https://doi.org/10.1145/142750.142769

Michael J Hove and Jane L Risen. 2009. It's all in the timing: Interpersonal synchrony increases affiliation. Social cognition 27, 6 (2009), 949-960.

Anya Kolesnichenko, Joshua McVeigh-Schultz, and Katherine Isbister. 2019. Understanding Emerging Design Practices for Avatar Systems in the Commercial Social VR Ecology. In Proceedings of the 2019 on Designing Interactive Systems Conference. ACM, 241-252.

Lucas Kovar, Michael Gleicher, and Frédéric Pighin. 2008. Motion Graphs. In ACM SIGGRAPH 2008 Classes (SIGGRAPH '08). ACM, New York, NY, USA, 51:1-51:10. https://doi.org/10.1145/1401132.1401202

Alexander Kulik, André Kunert, Stephan Beck, Carl-Feofan Matthes, Andre Schollmeyer, Adrian Kreskowski, Bernd Fröhlich, Sue Cobb, and Mirabelle D’Cruz. 2018. Virtual Valcamonica: collaborative exploration of prehistoric petroglyphs and their surrounding environment in multi-user virtual reality. PRESENCE: Teleoperators and Virtual Environments 26, 03 (2018), 297-321.

Jessica L. Lakin, Valerie E. Jefferis, Clara Michelle Cheng, and Tanya L. Chartrand. 2003. The chameleon effect as social glue: Evidence for the evolutionary significance of nonconscious mimicry. 7. nonverbal behavior 27, 3 (2003), 145-162. 
Marc Erich Latoschik, Florian Kern, Jan-Philipp Stauffert, Andrea Bartl, Mario Botsch, and Jean-Luc Lugrin. 2019. Not Alone Here?! Scalability and User Experience of Embodied Ambient Crowds in Distributed Social Virtual Reality. IEEE transactions on visualization and computer graphics 25, 5 (2019), 2134-2144.

Marc Erich Latoschik, Daniel Roth, Dominik Gall, Jascha Achenbach, Thomas Waltemate, and Mario Botsch. 2017. The Effect of Avatar Realism in Immersive Social Virtual Realities. In Proceedings of the 23rd ACM Symposium on Virtual Reality Software and Technology (VRST '17). ACM, New York, NY, USA, Article 39, 10 pages. https://doi.org/10.1145/3139131.3139156

A. Lecuyer, F. Lotte, R.B. Reilly, R. Leeb, M. Hirose, and M. Slater. 2008. Brain-Computer Interfaces, Virtual Reality, and Videogames. Computer 41, 10 (Oct. 2008), 66-72. https://doi.org/10.1109/MC.2008.410

Jehee Lee, Jinxiang Chai, Paul SA Reitsma, Jessica K. Hodgins, and Nancy S. Pollard 2002 b. Interactive control of avatars animated with human motion data. In $A C M$ Transactions on Graphics (TOG), Vol. 21. ACM, 491-500.

Sooha Park Lee, Jeremy B. Badler, and Norman I. Badler. 2002a. Eyes Alive. In $A C M$ Transactions on Graphics (TOG), Vol. 21. ACM, 637-644.

Hao Li, Laura Trutoiu, Kyle Olszewski, Lingyu Wei, Tristan Trutna, Pei-Lun Hsieh Aaron Nicholls, and Chongyang Ma. 2015. Facial performance sensing headmounted display. ACM Transactions on Graphics (TOG) 34, 4 (2015), 47

Simon Liversedge, Iain Gilchrist, and Stefan Everling. 2011. The Oxford handbook of eye movements. Oxford University Press.

Andrew Maimone and Henry Fuchs. 2011. A first look at a telepresence system with room-sized real-time $3 \mathrm{~d}$ capture and life-sized tracked display wall. Proceedings of ICAT 2011 (2011), 4-9.

Marvin Minsky. 1980. Telepresence. (1980).

Arjun Nagendran, Remo Pillat, Adam Kavanaugh, Greg Welch, and Charles Hughes 2014. A unified framework for individualized avatar-based interactions. Presence Teleoperators and Virtual Environments 23, 2 (2014), 109-132.

Soo Youn Oh, Jeremy Bailenson, Nicole Krämer, and Benjamin Li. 2016. Let the Avatar Brighten Your Smile: Effects of Enhancing Facial Expressions in Virtual Environments. PLOS ONE 11, 9 (09 2016), 1-18. https://doi.org/10.1371/journal. pone. 0161794

OptiTrack, Inc. 2018. Flex 3 - Technical Specifications for the Flex 3. http://optitrack com/products/flex-3/specs.html

Sergio Orts-Escolano, Christoph Rhemann, Sean Fanello, Wayne Chang, Adarsh Kowdle, Yury Degtyarev, David Kim, Philip L Davidson, Sameh Khamis, Mingsong Dou, et al. 2016. Holoportation: Virtual 3d teleportation in real-time. In Proceedings of the 29th Annual Symposium on User Interface Software and Technology. ACM, 741-754.

Kazuhiro Otsuka. 2016. MMSpace: Kinetically-augmented telepresence for small group-to-group conversations. In 2016 IEEE Virtual Reality (VR). 19-28.

Thammathip Piumsomboon, Gun A. Lee, Jonathon D. Hart, Barrett Ens, Robert W Lindeman, Bruce H. Thomas, and Mark Billinghurst. 2018. Mini-Me: An Adaptive Avatar for Mixed Reality Remote Collaboration. In Proceedings of the $2018 \mathrm{CH}$ Conference on Human Factors in Computing Systems (CHI '18). ACM, New York, NY, USA, Article 46, 13 pages. https://doi.org/10.1145/3173574.3173620

David Roberts, Robin Wolff, John Rae, Anthony Steed, Rob Aspin, Moira McIntyre, Adriana Pena, Oyewole Oyekoya, and Will Steptoe. 2009. Communicating eye-gaze across a distance: Comparing an eye-gaze enabled immersive collaborative virtual environment, aligned video conferencing, and being together. In 2009 IEEE Virtual Reality Conference. IEEE, 135-142. 00026.

Charles Rose, Brian Guenter, Bobby Bodenheimer, and Michael F. Cohen. 1996. Efficient generation of motion transitions using spacetime constraints. In Proceedings of the 23rd annual conference on Computer graphics and interactive techniques. ACM, 147-154.

Daniel Roth, Constantin Kleinbeck, Tobias Feigl, Christopher Mutschler, and Marc Erich Latoschik. 2018a. Beyond Replication: Augmenting Social Behaviors in Multi-User Virtual Realities. In 2018 IEEE Conference on Virtual Reality and 3D User Interfaces (VR). 215-222. https://doi.org/10.1109/VR.2018.8447550

Daniel Roth, Peter Kullmann, Gary Bente, Dominik Gall, and Marc Erich Latoschik 2018b. Effects of Hybrid and Synthetic Social Gaze in Avatar-Mediated Interactions. In 2018 IEEE International Symposium on Mixed and Augmented Reality Adjunct (ISMAR-Adjunct). 103-108. https://doi.org/10.1109/ISMAR-Adjunct.2018.00044

Daniel Roth, Marc Erich Latoschik, Kai Vogeley, and Gary Bente. 2015. Hybrid AvatarAgent Technology-A Conceptual Step Towards Mediated "Social" Virtual Reality and its Respective Challenges. $i$-com 14, 2 (2015), 107-114. https://doi.org/10.1515/ icom-2015-0030

Daniel Roth, Jean-Luc Lugrin, Julia Büser, Gary Bente, Arnulph Fuhrmann, and Marc Erich Latoschik. 2016a. A simplified inverse kinematic approach for embodied VR applications. In 2016 IEEE Virtual Reality (VR). 275-276. https: //doi.org/10.1109/VR.2016.7504760

Daniel Roth, Jean-Luc Lugrin, Dmitri Galakhov, Arvid Hofmann, Gary Bente, Marc Erich Latoschik, and Arnulph Fuhrmann. 2016b. Avatar realism and social interaction quality in virtual reality. In 2016 IEEE Virtual Reality (VR). 277-278. https://doi.org/10.1109/VR.2016.7504761

Daniel Roth, David Mal, Christian Felix Purps, Peter Kullmann, and Marc Erich Latoschik. 2018c. Injecting Nonverbal Mimicry with Hybrid Avatar-Agent Technologies: A Naïve Approach. In Proceedings of the Symposium on Spatial User Interaction
(SUI '18). ACM, New York, NY, USA, 69-73. https://doi.org/10.1145/3267782.3267791

Daniel Roth, Kristoffer Waldow, Felix Stetter, Gary Bente, Marc Erich Latoschik, and Arnulph Fuhrmann. 2016c. SIAMC: A Socially Immersive Avatar Mediated Communication Platform. In Proceedings of the 22nd ACM Conference on Virtual Reality Software and Technology (VRST). ACM, Munich, Germany, 357-358. https://doi.org/10.1145/2993369.2996302

Daniel Roth, Franziska Westermeier, Larissa Brübach, Tobias Feigl, Christian Schell, and Marc Erich Latoschik. 2019. Brain 2 Communicate: EEG-based Affect Recognition to Augment Virtual Social Interactions. (2019). https://doi.org/10.18420/muc2019ws- 571

Jamie Shotton, Toby Sharp, Alex Kipman, Andrew Fitzgibbon, Mark Finocchio, Andrew Blake, Mat Cook, and Richard Moore. 2013. Real-time human pose recognition in parts from single depth images. Commun. ACM 56, 1 (2013), 116-124.

Mel Slater. 2014. Grand Challenges in Virtual Environments. Frontiers in Robotics and AI 1 (2014), 3. https://doi.org/10.3389/frobt.2014.00003

Mel Slater, Bernhard Spanlang, Maria V. Sanchez-Vives, and Olaf Blanke. 2010. First Person Experience of Body Transfer in Virtual Reality. PLOS ONE 5, 5 (05 2010), 1-9. https://doi.org/10.1371/journal.pone.0010564

Mel Slater and Anthony Steed. 2002. Meeting People Virtually: Experiments in Shared Virtual Environments. In The Social Life of Avatars: Presence and Interaction in Shared Virtual Environments, Ralph Schroeder (Ed.). Springer London, London, 146-171. https://doi.org/10.1007/978-1-4471-0277-9 9

Harrison Jesse Smith and Michael Neff. 2018. Communication Behavior in Embodied Virtual Reality. In Proceedings of the 2018 CHI Conference on Human Factors in Computing Systems. ACM, 289. https://doi.org/10.1145/3173574.3173863

Oleg Špakov. 2012. Comparison of Eye Movement Filters Used in HCI. In Proceedings of the Symposium on Eye Tracking Research and Applications - ETRA '12. ACM Press, 281. https://doi.org/10.1145/2168556.2168616

Bernhard Spanlang, Jean-Marie Normand, David Borland, Konstantina Kilteni, Elias Giannopoulos, Ausiàs Pomés, Mar González-Franco, Daniel Perez-Marcos, Jorge Arroyo-Palacios, Xavi Navarro Muncunill, and Mel Slater. 2014. How to Build an Embodiment Lab: Achieving Body Representation Illusions in Virtual Reality. Frontiers in Robotics and AI 1 (2014), 9. https://doi.org/10.3389/frobt.2014.00009

Anthony Steed, William Steptoe, Wole Oyekoya, Fabrizio Pece, Tim Weyrich, Jan Kautz, Doron Friedman, Angelika Peer, Massimiliano Solazzi, Franco Tecchia, and others. 2012. Beaming: an asymmetric telepresence system. IEEE computer graphics and applications 32, 6 (2012), 10. https://doi.org/10.1109/MCG.2012.110

William Steptoe, Oyewole Oyekoya, Alessio Murgia, Robin Wolff, John Rae, Estefania Guimaraes, David Roberts, and Anthony Steed. 2009. Eye tracking for avatar eye gaze control during object-focused multiparty interaction in immersive collaborative virtual environments. In 2009 IEEE Virtual Reality Conference. IEEE, 83-90.

Evan A Suma, Belinda Lange, Albert Skip Rizzo, David M Krum, and Mark Bolas. 2011. Faast: The flexible action and articulated skeleton toolkit. In Virtual Reality Conference (VR), 2011 IEEE. IEEE, 247-248.

Unity Technologies. 2018. Unity - Manual: Preparing your own character. https://docs. unity3d.com/560/Documentation/Manual/Preparingacharacterfromscratch.html

Linda Tickle-Degnen and Robert Rosenthal. 1990. The Nature of Rapport and Its Nonverbal Correlates. Psychological Inquiry 1, 4 (1990), 285-293.

Michael Tomasello. 2009. Why we cooperate. MIT press.

Jolanda Tromp, Adrian Bullock, Anthony Steed, Amela Sadagic, Mel Slater, and Emmanuel Frécon. 1998. Small group behaviour experiments in the coven project. IEEE Computer Graphics and Applications 18, 6 (1998), 53-63.

Unity Technologies, ApS. 2018. Dissonance Voice Chat - Asset Store. https://assetstore.unity.com/packages/tools/audio/dissonance-voice-chat70078? aid=1100lJDF\&utm source $=$ aff

Alessandro Vinciarelli, Maja Pantic, and Hervé Bourlard. 2009. Social signal processing: Survey of an emerging domain. Image and vision computing 27, 12 (2009), 17431759.

Johannes Wagner, Florian Lingenfelser, Tobias Baur, Ionut Damian, Felix Kistler, and Elisabeth André. 2013. The social signal interpretation (SSI) framework: multimodal signal processing and recognition in real-time. In Proceedings of the 21st ACM international conference on Multimedia. ACM, 831-834.

Thomas Waltemate, Dominik Gall, Daniel Roth, Mario Botsch, and Marc Erich Latoschik. 2018. The Impact of Avatar Personalization and Immersion on Virtual Body Ownership, Presence, and Emotional Response. IEEE Transactions on Visualization and Computer Graphics 24, 4 (April 2018), 1643-1652. https: //doi.org/10.1109/TVCG.2018.2794629

L. Wilson and K. Seko. 2017-09. Head Mounted Display. Google Patents. https: //www.google.de/patents/US20170262703

Andrea Stevenson Won, Jeremy N Bailenson, and Jaron Lanier. 2015. Homuncular flexibility: the human ability to inhabit nonhuman avatars. Emerging Trends in the Social and Behavioral Sciences: An Interdisciplinary, Searchable, and Linkable Resource (2015), 1-16.

Jihun Yu and Jungwoon Park. 2017. Head-Mounted Display with Facial Expression Detecting Capability. Google Patents. https://www.google.com/patents/US20170091535 